\title{
Ho:YAG laser lithotripsy in non-contact mode: optimization of fiber to stone working distance to improve ablation efficiency
}

\author{
Vincent De Coninck ${ }^{1,2}$ (1) $\cdot$ Etienne Xavier Keller ${ }^{1,3} \cdot$ Paul Chiron $^{1} \cdot$ Laurian Dragos $^{4} \cdot$ Esteban Emiliani $^{5} \cdot$ Steeve Doizi $^{1}$. \\ Laurent Berthe $^{6}$. Olivier Traxer ${ }^{1}$
}

\begin{abstract}
Purpose To evaluate how variable working distances between the laser fiber and the stone influence ablation volume. Methods A laser fiber was fixed on a robotic arm perpendicular to an artificial stone. A single laser pulse was triggered at different working distances ( $0-2.0 \mathrm{~mm}$ in $0.2 \mathrm{~mm}$ increments) between the distal fiber tip and the stone. To achieve a measurable impact, pulse energy was set to 2 and $3 \mathrm{~J}$, with either short or long pulse duration. Ablation volume was calculated with an optical microscope. Experiments were repeated five times for each setting.

Results Highest ablation volume was observed with a long pulse of $3 \mathrm{~J}$ at a working distance of $0.4 \mathrm{~mm}$ between the laser fiber and the stone surface ( $p$ value $<0.05$ ). At $2 \mathrm{~J}$, the highest ablation volume was noticed with a short pulse in contact mode. However, ablation volume of the latter was not significantly greater than with a long pulse of $2 \mathrm{~J}$ at a working distance of $0.4 \mathrm{~mm}$ ( $p$ value $>0.05$ ). Compared to lithotripsy in contact mode, triggering a single long pulse at $0.4 \mathrm{~mm}$ increased ablation volume by $81 \%(p$ value $=0.016)$ at $2 \mathrm{~J}$ and by $89 \%(p$ value $=0.034)$ at $3 \mathrm{~J}$.

Conclusions For Ho:YAG laser lithotripsy, ablation volume may be higher in non-contact mode using long pulses, rather than in direct contact to the stone. Findings of the current study support the need of further studies of lithotripsy in noncontact mode.
\end{abstract}

Keywords Distance $\cdot$ Ho:YAG $\cdot$ Laser $\cdot$ Nephrolithiasis $\cdot$ Non-contact

\section{Abbreviation \\ Ho:YAG Holmium:yttrium-aluminum-garnet}

\section{Introduction}

Surgical procedures for kidney stones are performed very frequently due to their high prevalence. Generally, the holmium:yttrium-aluminum-garnet (Ho:YAG) laser generator is used when performing lithotripsy with a (flexible) ureteroscope. Until now, studies regarding in vitro laser

Vincent De Coninck and Etienne Xavier Keller contributed equally.

Electronic supplementary material The online version of this article (https://doi.org/10.1007/s00345-018-2589-8) contains supplementary material, which is available to authorized users.

Olivier Traxer

olivier.traxer@aphp.fr

Extended author information available on the last page of the article lithotripsy have focused on the effect of fragmentation efficiency by changing lithotripter settings and laser fiber diameters. Increasing pulse frequency, pulse energy or using short pulse durations results in increased stone ablation efficacy, retropulsion and fiber tip degradation [1-6]. Small-diameter fibers are as efficient as the larger-diameter counterparts but are more susceptible to fiber tip degradation [7]. This limitation is outweighed by the multiple benefits of lower retropulsion, better irrigation and better flexible scope deflection [3, 8]. Stripping the laser fiber tip significantly reduces ablation efficiency as well [9]. Regarding types of stones, laser lithotripsy of hard stones is associated with smaller ablation volumes, increased fiber tip degradation and longer operation time in comparison to soft stones [9-11].

Previously reported studies on laser efficiency have been performed with the laser fiber in direct contact with the stone surface. It is supposed that the smaller the distance between the laser fiber and the stone, the less energy is consumed during the formation of a vapor bubble and the greater amount of pulse energy remains for ablation [12]. Until now, no studies have been performed quantifying how variable 
working distances between the laser fiber tip and the stone influence fragmentation efficiency [13]. Better knowledge of laser lithotripsy in non-contact mode is crucial in the understanding of the working principle of Ho:YAG laser generators and to achieve optimal lithotripsy efficiency.

We aimed to evaluate how variable working distances between the laser fiber tip and the stone influence ablation volume, as a function of delivered energy and pulse duration. As a secondary objective, we aimed to define the influence of the holmium laser-induced vapor bubble on the ablation volume, as a function of the working distance.

\section{Materials and methods}

We used a $100 \mathrm{MHz}$ Synthesized Arbitrary Waveform Generator (model 395, Wavetek, UK) for controlling the laser to operate in single pulse mode and for synchronization of a single laser pulse with a high-speed camera. This generator was connected to a Ho:YAG laser machine (wavelength of $2100 \mathrm{~nm}$, maximum power output of $30 \mathrm{~W}$, Rocamed, Monaco) and to a high-speed camera (APX-RS 3000, Photron, San Diego, CA, USA) with magnifying lenses (3.0×, f/4, Edmund Optics, York, UK). High-speed imaging was performed at 12,000 frames (temporal resolution is $\sim 83 \mu \mathrm{s}$ ) and with a spatial resolution of $\sim 10 \mu \mathrm{m}$. A high-power lamp illuminated the laser fiber and stone to produce sufficient contrast between the $0.9 \%$ saline and the laser-induced bubbles. Activating the generator triggered a single laser pulse with a short delay in order that the entire bubble was recorded (Fig. 1).

We used artificial "hard" stones (BegoStone plus ${ }^{\circledR}$, powder to water ratio 5:1) to simulate a calcium oxalate monohydrate calculus in tensile strength. To produce homogeneous stones with a flat surface and free from air bubbles, we used a vibrating table, an electronically controlled vacuum mixing machine that also calibrated the powder-water ratio and Plexiglas molds $(24 \times 20 \times 18 \mathrm{~mm})$. Before lithotripsy, stones were examined for irregularities with micro-computed tomography.

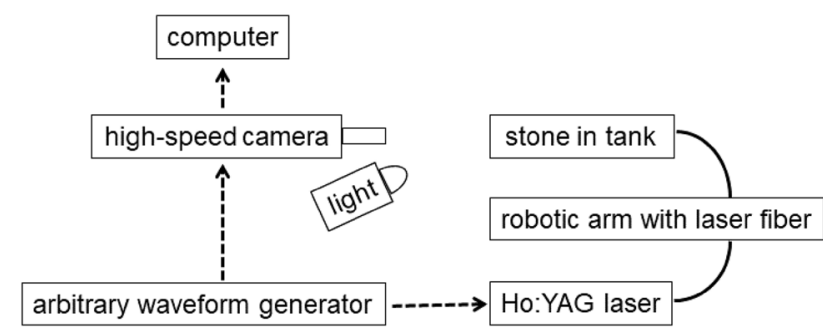

Fig. 1 Experimental setup for delivering a single Ho:YAG laser pulse at various working distances, recorded by a high-speed camera for imaging of cavitation bubbles
Using a support assembly with a robotic arm, a 272- $\mu \mathrm{m}$ core silica optical fiber (Rocamed, Monaco) was fixed perpendicular to the artificial stone. This robotic arm could be moved in a vertical plane with steps of $0.01 \mathrm{~mm}$. The stone and laser fiber tip were immersed in a tank filled with $0.9 \%$ saline at $25{ }^{\circ} \mathrm{C}$ for one hour prior to lithotripsy. The stone was not fixed in the tank but was not able to move due to its volume $\left(8640 \mathrm{~mm}^{3}\right)$. The laser fiber tip was cleaved before each experiment with metallic scissors and ten laser pulses were delivered in saline before delivering on the stone surface.

A preliminary study utilizing a single pulse at several distances showed a measurable impact starting from an energy of $2 \mathrm{~J}$. Therefore, single pulse energies of $2 \mathrm{~J}$ and $3 \mathrm{~J}$ were studied, with short $(150 \mu \mathrm{s})$ and long $(850 \mu \mathrm{s})$ pulse durations. These pulse durations were measured with a highspeed photodetector and a high-bandwidth oscilloscope. Fragmentation efficiency was tested for varying distances between the laser fiber tip and the stone: in contact, at 0.2 , $0.4,0.6,0.8,1.0,1.2,1.4,1.6,1.8$ and $2.0 \mathrm{~mm}$. To achieve statistically significant data, tests were repeated 5 times for each setting.

Each test created an ablation crater in the form of a cone. After drying the stone, the ablation volume was calculated with the formula of a cone. The ablation surface and depth were measured with a calibrated optical microscope (Axio Imager.M2m, Zeiss, Germany). Image processing software (Axiovision SE64, Zeiss, Germany) was used to calculate the ablation area in a plane of stone surface after outlining the outer borders of the impacted surface.

Continuous variables were reported as mean \pm standard deviation and were compared by unpaired Student's $t$ tests. For all tests, a two-sided $p$ value $<0.05$ was considered as statistically significant. All statistical tests were performed with GraphPad Prism 6.01 (GraphPad Software, La Jolla CA, USA).

\section{Results}

The highest ablation volume ( $p$ value $<0.05$ ) was observed with a long pulse of $3 \mathrm{~J}$ at a working distance of $0.4 \mathrm{~mm}$ between the laser fiber and the stone surface (Fig. 2). At $2 \mathrm{~J}$, the highest ablation volume was noticed with a short pulse in contact mode. However, ablation volume of the latter was not significantly greater than with a long pulse of $2 \mathrm{~J}$ at a working distance of $0.4 \mathrm{~mm}$ ( $p$ value $>0.05$ ).

Compared to lithotripsy in contact mode, triggering a single long pulse at $0.4 \mathrm{~mm}$ increased ablation volume by $81 \%$ $(p$ value $=0.016)$ at $2 \mathrm{~J}$ and by $89 \%(p$ value $=0.034)$ at $3 \mathrm{~J}$ (Table 1). At $0.4 \mathrm{~mm}$, both increasing energy from 2 to $3 \mathrm{~J}$ and increasing pulse duration from 150 to $850 \mu$ s increased stone ablation volume $(p$ value $<0.05)$ (Table 2$)$. Further 

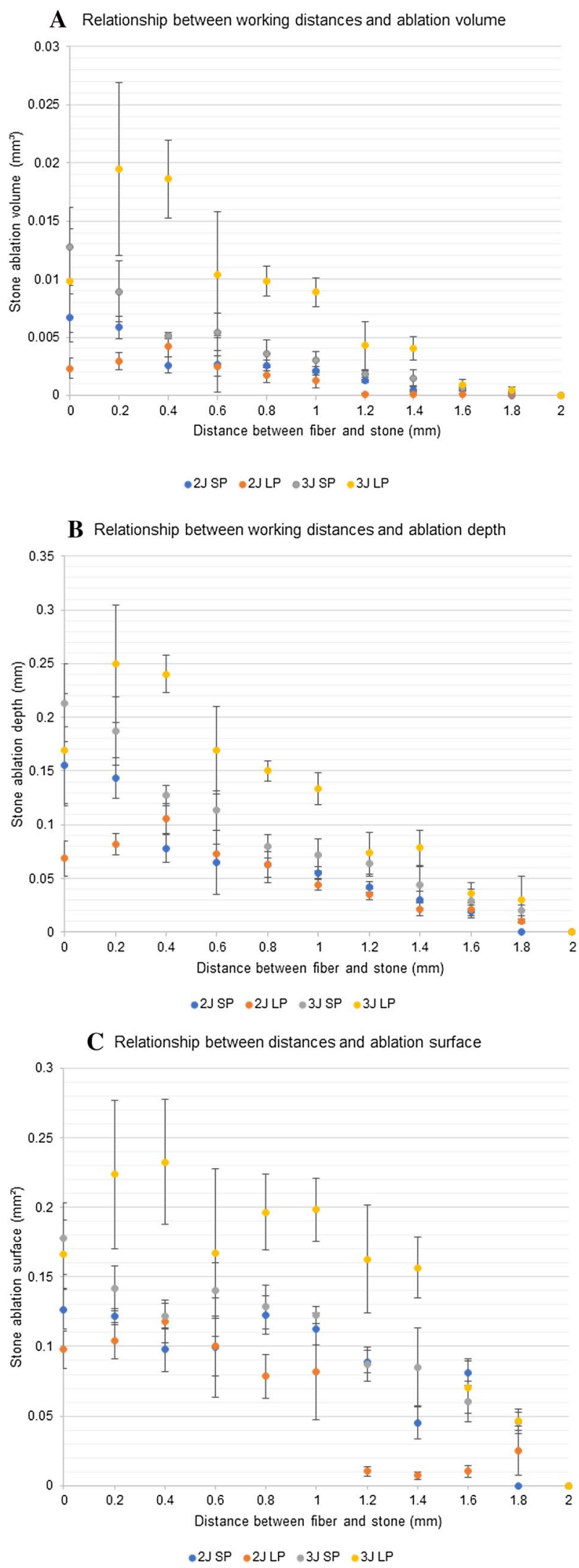

Fig. 2 Relationship of working distance on ablation volume (a), ablation depth (b) and ablation surface (c). $L P$ long pulse duration, $S P$ short pulse duration increasing working distances resulted in decreased ablation volumes, until no ablative effect was detected at $2.0 \mathrm{~mm}$.

In contact mode, increasing energy from 2 to $3 \mathrm{~J}$ resulted in mean increase of stone ablation volume by $92 \%$ $(p=0.028)$ using a short pulse and by $325 \%(p=0.006)$ using a long pulse (Table 2). In contact mode at $2 \mathrm{~J}$, stone ablation volume was $65 \%$ higher for a short pulse compared to a long pulse ( $p$ value $=0.004)$. At $3 \mathrm{~J}$, there was no significant difference in ablation volume between pulse durations $(p$ value $=0.336)$ in contact mode.

For long pulses, increasing working distances from contact mode to $0.4 \mathrm{~mm}$ increased stone ablation depth by $55 \%$ $(p$ value $=0.007)$ and by $47 \%(p$ value $=0.017)$ for $2 \mathrm{~J}$ and $3 \mathrm{~J}$, respectively. Increasing the working distance further decreased ablation depth, until no ablative effect was detected at $2.0 \mathrm{~mm}$ (Fig. 2). For short pulses, ablation depth was deepest in contact mode and decreased as the distance between the laser and the fiber increased. The ablation surface was greatest at $0.4 \mathrm{~mm}$ for long pulses and in contact mode for short pulses.

In Fig. 3 and Supplementary material 1 and 2, the evolution of the cavitation bubble for a long and short pulse is displayed. A long pulse trigger produced a pear-shaped smaller and shorter bubble, characterized by a chaotic multiple bubble stream, each locally enlarging and collapsing apart from the other bubbles. These dynamics were noticed at all examined working distances. The bubble from a short pulse was characterized by the formation of a spherical shaped wide and long bubble, followed by a collapse, without a chaotic multiple bubble stream.

\section{Discussion}

Single-pulse lithotripsy was most efficient at $0.4 \mathrm{~mm}$ using long pulses at $3 \mathrm{~J}$. For lithotripsy with a working distance of $0.4 \mathrm{~mm}$, increasing pulse duration from 150 to $850 \mu \mathrm{s}$ increased ablation volume significantly for examined energies. Increasing working distances further gradually decreased ablation volume, ablation depth and ablation surface. To our knowledge, the added value of laser lithotripsy in non-contact mode on ablation volume is reported for the first time. The only previous studies that are considered as non-contact lithotripsy were concerning the popcorn technique $[14,15]$. In comparison to our results, these authors found the highest efficiency with high energy and long pulse durations. In contrast, other authors always stated that Ho:YAG laser lithotripsy is most efficient in contact mode [16, 17]. However, these statements were based on a few non-contact mode experiments with a very low energy $(0.375 \mathrm{~J})$ and a relative long working distance $(1 \mathrm{~mm})$ between the laser fiber and the stone surface. 
Table 1 Effect of contact and non-contact single pulse lithotripsy on stone ablation volume (in $\mathrm{mm}^{3}$ ), depth (in $\mathrm{mm}$ ) and surface (in $\mathrm{mm}^{2}$ ) \pm standard deviation, with respect to increase (\%) of distance from $0 \mathrm{~mm}$ to $0.4 \mathrm{~mm}$

\begin{tabular}{llllr}
\hline & Contact & $0.4 \mathrm{~mm}$ & Difference $\%$ & $p$ value \\
\hline Volume, mm ${ }^{3}$ & & & & \\
2 J SP & $0.0067 \pm 0.0021$ & $0.0026 \pm 0.0007$ & -61.0 & 0.010 \\
2 J LP & $0.0023 \pm 0.0009$ & $0.0042 \pm 0.0009$ & +81.5 & 0.016 \\
3 J SP & $0.0128 \pm 0.0034$ & $0.0051 \pm 0.0003$ & -59.9 & 0.001 \\
3 J LP & $0.0099 \pm 0.0044$ & $0.0186 \pm 0.0033$ & +88.7 & 0.034 \\
Depth, mm & & & & \\
2 J SP & $0.1557 \pm 0.0355$ & $0.0776 \pm 0.0130$ & -50.1 & 0.006 \\
2 J LP & $0.0685 \pm 0.0162$ & $0.1058 \pm 0.0142$ & +54.5 & 0.007 \\
3 J SP & $0.2134 \pm 0.0361$ & $0.1272 \pm 0.0091$ & -40.4 & 0.001 \\
3 J LP & $0.1698 \pm 0.0520$ & $0.2499 \pm 0.0172$ & +47.1 & \\
Surface, mm & & & & 0.079 \\
2 J SP & $0.1265 \pm 0.0142$ & $0.0979 \pm 0.0158$ & -22.6 & 0.101 \\
2 J LP & $0.0977 \pm 0.0133$ & $0.1181 \pm 0.0156$ & +20.8 & 0.096 \\
3 J SP & $0.1776 \pm 0.0256$ & $0.1217 \pm 0.0090$ & -31.5 & 0.029 \\
3 J LP & $0.1662 \pm 0.0245$ & $0.2325 \pm 0.0449$ & +39.9 & \\
\hline
\end{tabular}

$L P$ long pulse duration, $S P$ short pulse duration

\begin{tabular}{|c|c|c|c|c|}
\hline & \multicolumn{2}{|c|}{ Ablation volume $\pm \mathrm{SD}$} & \multirow[t]{2}{*}{ Difference \% } & \multirow[t]{2}{*}{$p$ value } \\
\hline & $2 \mathrm{~J} \mathrm{SP}$ & $3 \mathrm{~J} \mathrm{SP}$ & & \\
\hline Contact & $0.0067 \pm 0.0021$ & $0.0128 \pm 0.0034$ & +91.6 & 0.028 \\
\hline \multirow[t]{2}{*}{$0.4 \mathrm{~mm}$} & $0.0026 \pm 0.0007$ & $0.0051 \pm 0.0003$ & +97.1 & $<0.001$ \\
\hline & $2 \mathrm{~J} \mathrm{LP}$ & $3 \mathrm{~J} \mathrm{LP}$ & & \\
\hline Contact & $0.0023 \pm 0.0009$ & $0.0099 \pm 0.0044$ & +325.3 & 0.006 \\
\hline \multirow[t]{2}{*}{$0.4 \mathrm{~mm}$} & $0.0042 \pm 0.0009$ & $0.0186 \pm 0.0033$ & +342.4 & $<0.001$ \\
\hline & $2 \mathrm{~J} \mathrm{SP}$ & $2 \mathrm{~J} \mathrm{LP}$ & & \\
\hline Contact & $0.0067 \pm 0.0021$ & $0.0023 \pm 0.0009$ & -65.3 & 0.004 \\
\hline \multirow[t]{2}{*}{$0.4 \mathrm{~mm}$} & $0.0026 \pm 0.0007$ & $0.0042 \pm 0.0009$ & +61.7 & 0.037 \\
\hline & $3 \mathrm{~J} \mathrm{SP}$ & $3 \mathrm{~J} \mathrm{LP}$ & & \\
\hline Contact & $0.0128 \pm 0.0034$ & $0.0099 \pm 0.0044$ & -22.9 & 0.336 \\
\hline $0.4 \mathrm{~mm}$ & $0.0051 \pm 0.0003$ & $0.0186 \pm 0.0033$ & +263.0 & $<0.001$ \\
\hline
\end{tabular}

$L P$ long pulse duration, $S P$ short pulse duration
Table 2 Effect of increasing energy or pulse duration on stone ablation volume (in $\left.\mathrm{mm}^{3}\right) \pm$ standard deviation, with respect to increase (\%) of energy (from 2 to $3 \mathrm{~J}$ ) or pulse duration (from 150 to $850 \mu \mathrm{s}$ )
Furthermore, studying non-contact laser lithotripsy efficiency was never a primary endpoint in these studies.

Currently, it is unclear why ablation volume with a long Ho:YAG laser pulse is highest in non-contact mode. Since holmium bubbles are necessary for the thermomechanical contribution to laser ablation [18], the presence of the micro-bubble stream as noticed with the high-speed camera for long pulses may play a role in the ablative effect, as well as the shielding effect of products of ablation during the pulse, and the laser beam scattering and divergence. The vapor channel of the long pulse, characterized by the pear-shaped bubble and chaotic multiple bubble stream, is probably most efficient to conduct the laser energy directly onto the stone surface at a distance of $0.4 \mathrm{~mm}$. Interestingly, the best results are for a working distance of $0.4 \mathrm{~mm}$ which correlates exactly with the optical penetration depth of Ho:YAG laser energy in water. However, this may just be a coincidence.

In contact mode at $3 \mathrm{~J}$, there was no difference in ablation volume between short and long pulses. This observation is consistent with previous reports by Sroka et al. studying the impact of pulse durations on Ho:YAG laser lithotripsy [19]. For lower energies, a short pulse resulted in a higher ablation volume compared to a long pulse in contact mode. This was also reported by Wezel et al. using the same energy [20].

In contrast to the report by Sroka et al. studying singlepulse Ho:YAG laser lithotripsy, mean volumes of our craters were smaller [19]. This may be explained by the fact that our stones had a higher powder-water ratio and were free from air bubbles using a vibrating table and an electronically controlled vacuum mixing machine. Moreover, the energy level of the first pulse was most likely not the 

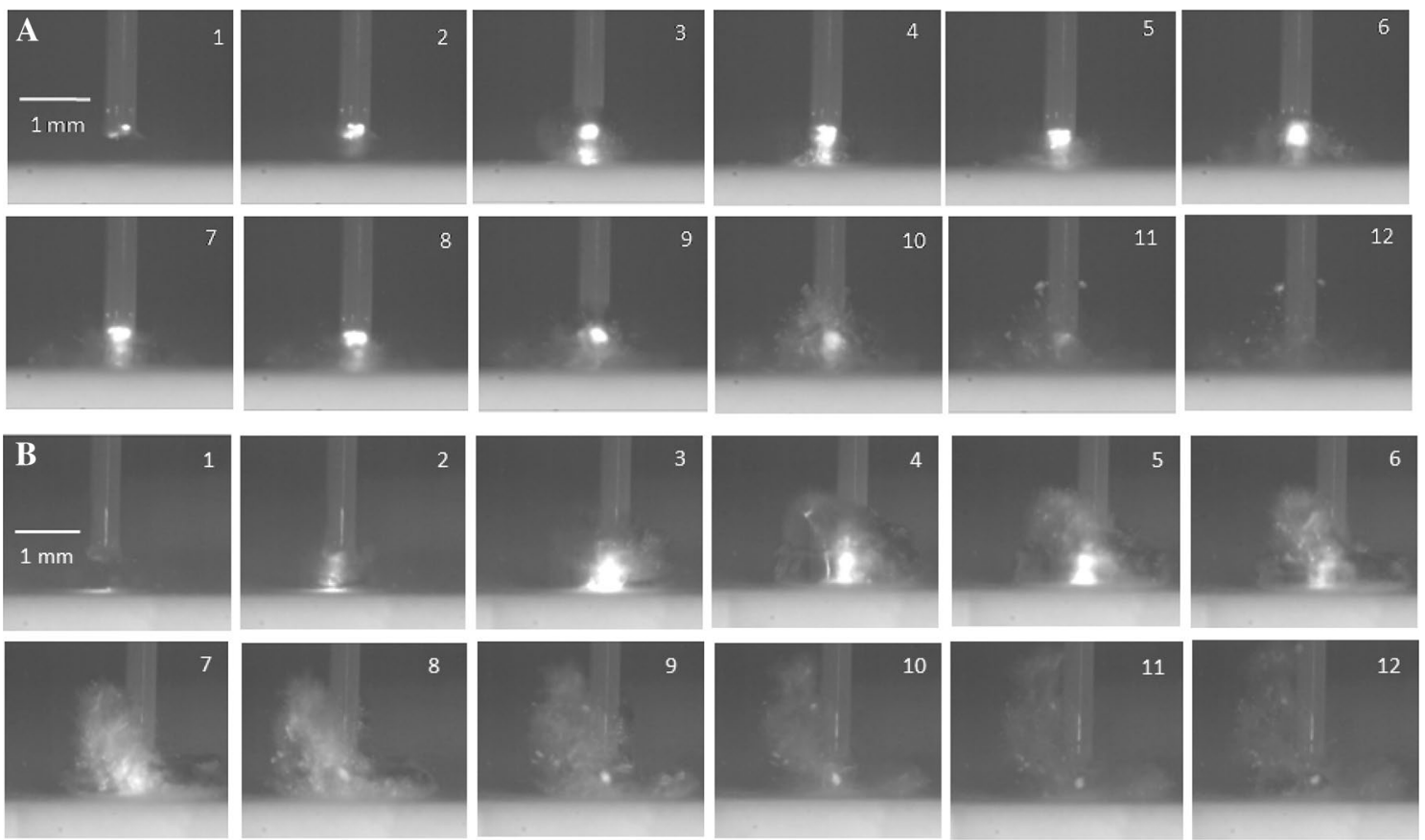

Fig. 3 Frames taken with the high-speed camera showing the formation of bubble expansions and collapses with a $272 \mu \mathrm{m}$ core silica optical fiber at $2 \mathrm{~J}$ at a working distance of $0.4 \mathrm{~mm}$ at successive intervals of $83 \mu$ s during a pulse duration of $850 \mu \mathrm{s}(\mathbf{a})$ and $150 \mu \mathrm{s}(\mathbf{b})$

expected energy level since it tends to be more stable after a couple of pulses. As well, cutting a laser fiber with metallic scissors decreases power by $30 \%$ [21]. To avoid that fiber coating remnants would influence the energy by covering the laser fiber tip, ten laser pulses were delivered in saline before delivering on the stone surface. Our study has several limitations. First, artificial stones are not an ideal substitute for human stones. However, to produce homogeneous stones with a flat surface and free from air bubbles, artificial stones were produced with the greatest precision by a vibrating table, an electronically controlled vacuum mixing machine, followed by micro-computed tomography control before experiments. Second, ablation volumes were calculated by the formula of a cone. However, craters were not perfectly shaped cones. To prevent potential variations in interpretation of the data between investigators, a single author (VDC) performed all measurements. The low standard deviations demonstrate a sufficient standardization and reproducibility. Third, we only took the pulse energy and duration into account when examining ablation volume of single pulses. We preferred not to include frequency, since repetitive delivery of a train of laser pulses leads to formation of a crater, which alters the initial distance between the laser fiber tip and the stone. Since we used the first pulse delivered by the laser generator, this may be considered as being a possible source of variability, because energy level usually tends to be more stable after a couple of pulses. Finally, we only examined laser energies of $2 \mathrm{~J}$ and $3 \mathrm{~J}$, since lower energies prevented precise microscopic evaluation. However, such high energies are seldom used in clinical practice during lithotripsy. With these results, we want to draw attention that lithotripsy in non-contact mode may be more efficient than in contact mode, rather than stating that lithotripsy should be performed at energies of $2 \mathrm{~J}$ or higher.

Besides pulse duration, energy and frequency, the distance between the laser fiber tip and the stone appears to be a fourth parameter that may improve lithotripsy efficiency. However, future studies are necessary to confirm these findings. These experiments should be performed with lower energies ranging from 0.2 to $1.0 \mathrm{~J}$ that are typically used during laser lithotripsy procedures. As well, the influence of different fiber sizes, fiber distal end conditions, different pulse repetition rates, shapes of laser pulse (including Moses technology), and types of stones should be studied. It would also be interesting to evaluate the impact of lithotripsy in non-contact mode on retropulsion. To approach clinical situations, stone ablation volume should also be evaluated with a moving laser fiber for different frequencies at several fiber working distances. Once the ideal distance per laser settings are set, this may lead to new developments. Since specific distances are difficult to maintain during surgical intervention, this may be overcome by the development of laser fibers with a specific buffer or lasers that only activate at specific fiber to stone working distances. Lithotripsy in non-contact mode may also be of interest for lower calix stones that are difficult to reach or stones that are not in 
the direct line of sight because of limitations of endoscope deflection. Understanding how distance stands in relation to various laser settings is essential to improve practice and treatment strategies of endourological stone management and to achieve optimal lithotripsy efficiency.

\section{Conclusion}

For Ho:YAG laser lithotripsy, ablation volume seems to increase in non-contact mode using long pulses, rather than in direct contact to the stone. The resulting increase in ablation volume may possibly be caused by a pear-shaped small and short bubble, characterized by a chaotic multiple bubble stream, each locally enlarging and collapsing apart from the other bubbles. Findings of the current study support the need of further studies of laser lithotripsy in non-contact mode.

Acknowledgements We would like to express our greatest appreciation to Prof. Gregory Altshuler, Prof. Nathaniel M. Fried and Viktoriya Vinnichenko for their valuable suggestions and proofreading of this article.

Author contributions VDC: protocol/project development, data collection or management, data analysis, manuscript writing/editing. EXK: protocol/project development, data collection or management, data analysis, manuscript writing/editing. PC: protocol/project development, data collection or management, data analysis. LD: protocol/ project development. EE: protocol/project development. SD: protocol/ project development, data analysis. LB: protocol/project development, data analysis. OT: protocol/project development, data collection or management, data analysis, manuscript writing/editing.

Funding Prof. Olivier Traxer is a consultant for Coloplast, Rocamed, Olympus, EMS and Boston Scientific. Dr. Steeve Doizi is a consultant for Coloplast. Dr. Vincent De Coninck is supported by the EUSP scholarship from the European Association of Urology and by a grant from the Belgische Vereniging voor Urologie (BVU). Dr. Etienne Xavier Keller is supported by a Travel Grant from the University Hospital Zurich and by a grant from the Kurt and Senta Herrmann Foundation.

\section{Compliance with ethical standards}

Ethical statement We did not perform research involving human participants and/or animals.

\section{References}

1. Kronenberg P, Traxer O (2015) Update on lasers in urology 2014: current assessment on holmium:yttrium-aluminium-garnet (Ho:YAG) laser lithotripter settings and laser fibers. World J Urol 33:463-469

2. Vassar GJ, Teichman JM, Glickman RD (1998) Holmium:YAG lithotripsy efficiency varies with energy density. J Urol 160:471-476
3. Kronenberg P, Traxer O (2014) In vitro fragmentation efficiency of holmium: yttrium-aluminium-garnet (YAG) laser lithotripsya comprehensive study encompassing different frequencies, pulse energies, total power levels and laser fibre diameters. BJU Int 114:261-267

4. Talso M, Emiliani E, Haddad M et al (2016) Laser fiber and flexible ureterorenoscopy: the safety distance concept. J Endourol 30:1269-1274

5. Kalra P, Le NB, Bagley D (2007) Effect of pulse width on object movement in vitro using holmium:YAG laser. J Endourol 21:228-231

6. Sea J, Jonat LM, Chew BH et al (2012) Optimal power settings for holmium:YAG LITHOTRIPSY. J Urol 187:914-919

7. Kronenberg P, Traxer O (2014) The truth about laser fiber diameters. Urology 84:1301-1307

8. Pasqui F, Dubosq F, Tchala K et al (2004) Impact on active scope deflection and irrigation flow of all endoscopic working tools during flexible ureteroscopy. Eur Urol 45:58-64

9. Kronenberg P, Traxer O (2015) Are we all doing it wrong? Influence of stripping and cleaving methods of laser fibers on laser lithotripsy performance. J Urol 193:1030-1035

10. Knudsen BE, Pedro R, Hinck B, Monga M (2011) Durability of reusable holmium:YAG laser fibers: a multicenter study. J Urol 185:160-163

11. Molina WR, Marchini GS, Pompeo A, Sehrt D, Kim FJ, Monga M (2014) Determinants of holmium:yttrium-aluminium-garnet laser time and energy during ureteroscopic laser lithotripsy. Urology 83:738-744

12. Lu T, Xiao Q, Xia D, Ruan K, Li Z (2010) Cavitation effect of holmium laser pulse applied to ablation of hard tissue underwater. J Biomed Opt 15:048002

13. De Coninck V, Keller EX, Chiron P, Doizi S, Traxer O (2018) Contact or Noncontact Laser Lithotripsy? (From: Tracey J, Gagin G, Morhardt D, et al. J Endourol 2018;32:290-295). J Endourol 32:987-988

14. Emiliani E, Talso M, Cho SY et al (2017) Optimal settings for the noncontact holmium:YAG stone fragmentation popcorn technique. J Urol 198:702-706

15. Chawla SN, Chang MF, Chang A, Lenoir J, Bagley DH (2008) Effectiveness of high-frequency holmium:YAG laser stone fragmentation: the "popcorn effect". J Endourol 22:645-650

16. Vassar GJ, Chan KF, Teichman JM et al (1999) Holmium: YAG lithotripsy: photothermal mechanism. J Endourol 13:181-190

17. Chan KF, Vassar GJ, Pfefer TJ et al (1999) Holmium:YAG laser lithotripsy: a dominant photothermal ablative mechanism with chemical decomposition of urinary calculi. Lasers Surg Med 25:22-37

18. Hardy LA, Kennedy JD, Wilson CR, Irby PB, Fried NM (2017) Analysis of thulium fiber laser induced bubble dynamics for ablation of kidney stones. J Biophoton 10:1240-1249

19. Sroka R, Pongratz T, Scheib G et al (2015) Impact of pulse duration on Ho:YAG laser lithotripsy: treatment aspects on the singlepulse level. World J Urol 33:479-485

20. Wezel F, Hacker A, Gross AJ, Michel MS, Bach T (2010) Effect of pulse energy, frequency and length on holmium:yttrium-aluminium-garnet laser fragmentation efficiency in non-floating artificial urinary calculi. J Endourol 24:1135-1140

21. Haddad M, Emiliani E, Rouchausse Y et al (2017) Impact of laser fiber tip cleavage on power output for ureteroscopy and stone treatment. World J Urol 35:1765-1770 


\title{
Affiliations
}

\section{Vincent De Coninck ${ }^{1,2}$ (1) $\cdot$ Etienne Xavier Keller ${ }^{1,3} \cdot$ Paul Chiron $^{1} \cdot$ Laurian Dragos $^{4} \cdot$ Esteban Emiliani $^{5} \cdot$ Steeve Doizi $^{1}$. Laurent Berthe $^{6}$. Olivier Traxer ${ }^{1}$}

\author{
Vincent De Coninck \\ vdconinck@gmail.com \\ Etienne Xavier Keller \\ etienne.xavier.keller@gmail.com \\ Paul Chiron \\ paul.chiron@intradef.gouv.fr \\ Laurian Dragos \\ lauriandragos@yahoo.com \\ Esteban Emiliani \\ emiliani@gmail.com \\ Steeve Doizi \\ steeve.doizi@aphp.fr \\ Laurent Berthe \\ laurent.berthe@ensam.eu
}

1 Sorbonne Université, GRC n ${ }^{\circ} 20$, Groupe de Recherche Clinique Sur La Lithiase Urinaire, AP-HP, Hôpital Tenon, 75020 Paris, France

2 Department of Urology, AZ Klina, Brasschaat, Belgium

3 Department of Urology, University Hospital Zurich, University of Zurich, Zurich, Switzerland

4 Department of Urology, University of Medicine and Pharmacy "Victor Babes" Timişoara, Timisoara, Romania

5 Fundació Puigvert, Department of Urology, Universidad Autonoma de Barcelona, Barcelona, Spain

6 Process and Engineering in Mechanics and Materials Laboratory (PIMM), UMR CNRS/ENSAM, Paris, France 